\title{
Local Impedance Spectroscopy: A Potential Tool to Characterize the Evolution of Emulsions and Foams
}

\author{
Michael Held ${ }^{1}$, Patrick Schwaller ${ }^{1}$, Markus Vaihinger ${ }^{2}$ and Christoph Denkel ${ }^{2}$ \\ 1. Institute for Applied Laser, Photonics and Surface Technologies ALPS, Bern University of Applied Sciences, Pestalozzistrasse 20, \\ Burgdorf CH-3400, Switzerland \\ 2. Division of Food Science and Management, Bern University of Applies Sciences, Länggasse 85, Zollikofen CH-3052, Switzerland
}

\begin{abstract}
The potential of local impedance spectroscopy (IS) to access changes in emulsions and foams has been investigated. As test systems we used the separation of a simple oil/vinegar mixture as well as the whipping process of dairy cream. For the latter, IS data were compared to particle size distribution (PSD) measurements. Our measurements show that local IS is indeed a valuable tool to locally study processes in emulsion. On one hand, it seems to be very sensitive to small water quantities in oil thus being a suitable method for process control in water removing processes. On the other hand, concerning fat foams, it seems to be able to detect the evolution of foam structures. Both examples show that local IS could be a helpful tool for process control.
\end{abstract}

Key words: Local IS, emulsions, foams, whipping of cream.

\section{Introduction}

There is a growing need for real-time characterization of food products to adjust or guarantee stable conditions during production and/or storage [1-4]. One promising approach to investigate non-invasively quality aspects and structural properties of food, in steady-state or under change, is impedance spectroscopy (IS), especially if properties can be investigated locally, i.e. on small sample volumes. IS is a versatile concept for the investigation and characterization of dielectric and conductive properties of materials. Therefore, in principle all material properties or property modifications influencing dielectric and conductive properties can be addressed by IS.

The technique is based on the measurement of impedance (i.e. the material's tendency to resist the flow of electrical charge) of a system, which was excited with an alternating current or an alternating

Corresponding author: Christoph Denkel, Dr. sc. ETH, Dipl.-Lm-Ing., research fields: food structure design, food processing 4.0, food process design, digital food production, individualization of food. voltage. The impedance spectrum is obtained by measuring the impedance of the sample over a wide frequency range, typically over several decades. Closely related methods to IS are conductometry and dielectric spectroscopy, which differ mainly in the ways of presenting results and the used frequency ranges or equipment [5].

Although the basics of IS have been known since the early 20th century, the first application in the field of food technology was reported only in 1975, where the dielectric properties of frozen fish has been measured [6]. Recently, IS has been used for nondestructive monitoring in different areas of food processing and analysis, for instance: estimation of meat maturation states [7, 8]; determination of the origin of unifloral honeys [9]; assessment of the quality of fruits [10]; detection of microbial concentration in ice-cream [11]; controlling ice-cream freezing [12]. These examples all have in common that macroscopic sample volumes were examined.

In this work we therefore address the issue to which extent IS with microscopic electrodes can be useful to study local properties of food products or emulsions. 
For this purpose we investigated two different model systems: An oil-vinegar emulsion was used to investigate whether the phase separation of the mixture can be monitored in quasi-real time. As a second system we recorded local IS signals for different states of the whipping process of dairy cream and correlated the obtained results with measurements of the cream's particle size distribution (PSD) as measured by light scattering.

\section{Materials and Methods}

\subsection{Samples}

Two types of samples were examined by local IS. To observe the phase separation by IS, ordinary sunflower oil and white wine vinegar were mixed in a $1: 1$ ratio by gentle agitation. As part of the phase separation process of the oil-water-emulsion, very likely coalescence plays a role. The IS hereby measures the superposition of the different effects. It is not straightforward to separate the different contributions but this is not the focus for the measurements presented below. We have chosen the oil-vinegar emulsion because of its easy handling perfectly suited for testing the measurement device. Simple storage, room temperature measurements are possible, the phase separation can be easily observed by eye and happens on a well-suited time scale but still within a rather localized volume. Coalescence could simply be prevented by the addition of emulsifiers in combination with intensive emulsification. However, for the time being we preferred to keep the experimental setup very simple and to have a quick optical control due to fast phase separation over the sample. The temperature of the equipment and the emulsion were stabilized at room temperature. Measurements were started immediately after the mixing procedure.

As a second specimen, organic pasteurized, non-homogenized dairy cream $(100 \mathrm{~mL}$, standardized fat content of $35 \%$, no added stabilizers), obtained from a local supermarket has been chosen to monitor the different whipping stages by IS. The samples were whipped in the cream tester for different time intervals until the desired PE-value (between a minimum of 25 and a maximum of 48 ; number of the apparatus to indicate beating resistance of the evolving foam) was reached. For each of these cream samples the PSD was measured and IS was performed. The bipolar electrode was placed in the sample immediately after the interruption of the whipping process in always the same manner. For each sample of whipped cream the IS as a function of the PE-value was measured four times. The temperature of the samples and the whipping equipment were kept constant at $5 \pm 1{ }^{\circ} \mathrm{C}$. A constant temperature during the cream measurements is very important because significant changes of the impedance have been observed as a consequence of even minor temperature changes. Before each measurement, the sensor was washed in a diluted detergent, rinsed with ultra-pure water for at least 5 min and finally dried by exposure to a flow of dry and oil-free compressed air.

\subsection{Measurement Devices}

\subsubsection{Impedance Spectroscopy}

In a typical impedance measurement, a sinusoidal current $(t)$ with angular frequency $\omega$ passed through a sample. In the steady state, the voltage drop $u(t)$ over the sample is also sinusoidal with angular frequency $\omega$, but may be shifted in phase $\varphi$ in relation to the current. According to Ohm's Law, the impedance $\mathrm{Z}$ can be calculated as the ratio $u(t)$ to $i(t)$ :

$$
Z=\frac{u(t)}{i(t)}=\frac{U_{0} e^{j\left(\omega t+\varphi_{U}\right)}}{I_{0} e^{j\left(\omega t+\varphi_{I}\right)}}=\frac{U_{0}}{I_{0}} e^{j\left(\varphi_{U}-\varphi_{I}\right)}=|Z| e^{j \varphi}(1)
$$

The complex impedance $\mathrm{Z}$ can be graphically represented in a vector diagram as real and imaginary part or in the polar coordinate system as absolute value and phase angle. If we do not only use a single frequency for exciting the sample, but many different frequencies in a frequency range, we obtain the impedance spectrum of the system. A popular presentation method for the impedance spectrum is the 
so called Bode Plot as shown in Fig. 1.

The frequency is assigned on a logarithmic scale to the horizontal axis, while the absolute values of the impedance (logarithmic scale) and the phase shift (linear scale) are plotted on the vertical axes. In Fig. 1, the impedance and phase shift values of a simple $\mathrm{RC}$-circuit $(\mathrm{R}=$ resistance, $\mathrm{C}=$ capacitor) are shown as an illustrative example.

\subsubsection{Local Impedance Spectroscopy}

Local IS works in principle as described above, however the probed volume is reduced in our case to about $1 \mathrm{~mm}^{3}$. As a consequence, microscopic electrodes have to be used and one has to deal with possible boundary effects and/or modifications of the surface properties of the microscopic electrodes. In return, such local measurements allow addressing processes like the phase separation of oil and vinegar where the interface zone is thin, or the whipping process of cream where particles with diameters below $100 \mu \mathrm{m}$ play a role. As already mentioned, the miniaturization involves also some disadvantages and challenges. In particular we would like to point to the increasing influence of parasitic elements with decreasing electrode surface. In addition, the electrode polarization and the nonlinear behavior of the electrode at high exiting voltages play an important role. Guidelines for considering such effects can be found in the literature (Kohlrausch's Law, [13]).
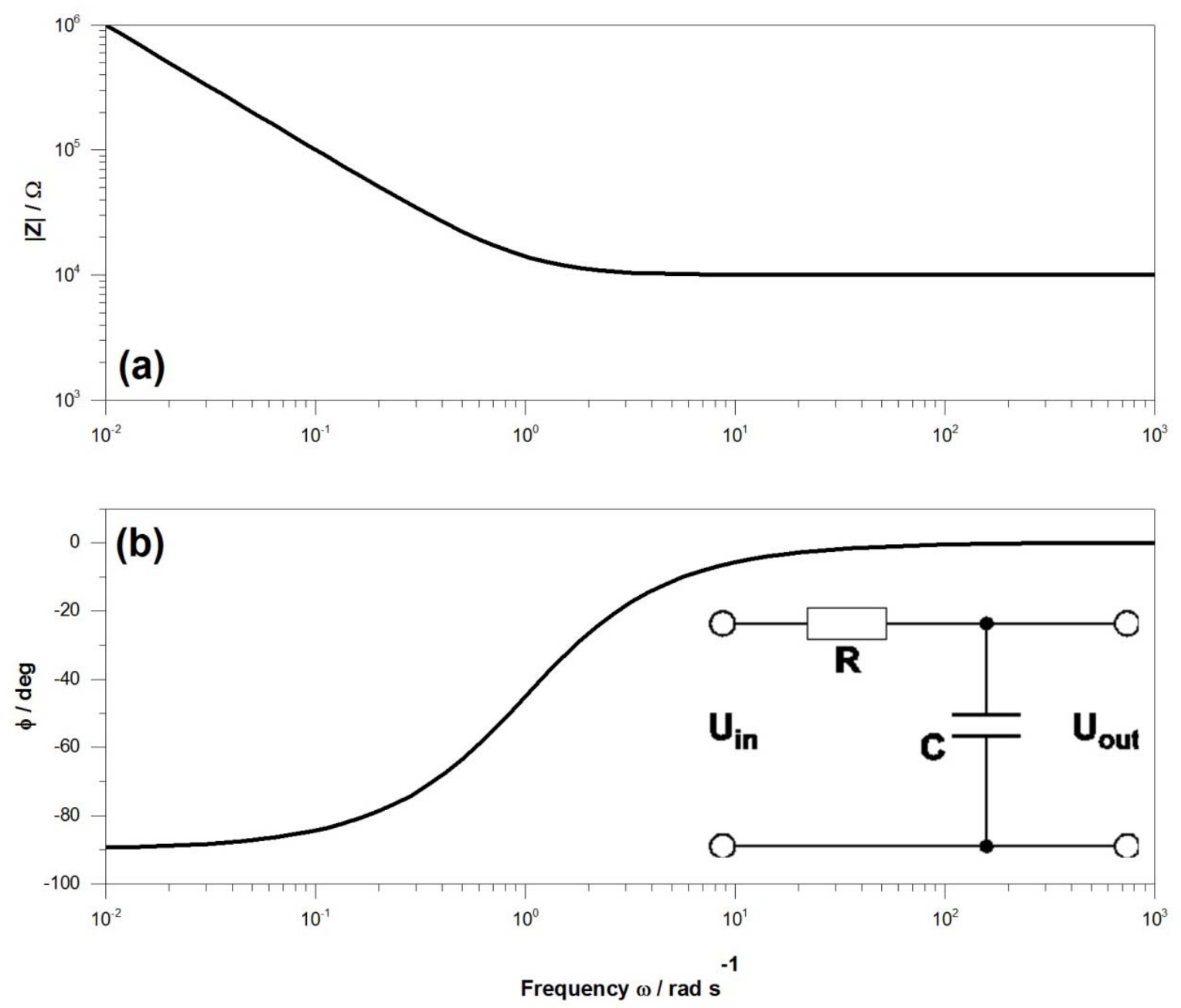

Fig. 1 Impedance spectrum of an $R C$-circuit $(R=10 \mathrm{k} \Omega$ and $C=100 \mu F)$ : (a) absolute values of the impedance; (b) phase shift. The inset in the lower graph shows the corresponding electrical circuit. 


\subsubsection{Electrode Design}

In order to perform IS on a sample, at least one pair of electrodes (referred as two-electrode or bipolar method) is required. The sinusoidal current $\mathrm{i}(\mathrm{t})$ is injected into the sample through two electrical contacts and the resulting voltage drop $u(t)$ is measured between the same two contacts. The two-electrode method works properly if the impedance of the electrode-electrolyte interface is much lower than the impedance of the sample since the measured impedance is the sum of both terms [14]. Above approximately $1,000 \mathrm{~Hz}$ the electrode-electrolyte interface impedance is dominated by the electrolyte resistance, where we can readily use the bipolar method above 1,000 Hz. The electrode setup for the measurements presented in this work is shown in Fig. 2. It consists of two gold electrodes $1,200 \mu \mathrm{m} \times 500 \mu \mathrm{m}$, separated by a $200 \mu \mathrm{m}$ wide gap (Fig. 3). The electrodes (thickness of the Au layer 133 $\pm 2 \mathrm{~nm}$ as measured with a stylus profilometer) were deposited on a glass substrate by physical vapor deposition and patterned by standard lithography and etching processes. The leads have been passivated by polyimide tape (3M, Maplewood, Minnesota, U.S.). The electrodes were connected in four-wire configuration with coaxial cables to the precision LCR meter HP 4284A to cancel out resistance errors of the leads. Furthermore, calibration measurements were carried out on the measuring system, which are intended to eliminate automatically the parasitic effects caused by the test leads. The impedance analyzer scanned 48 frequencies distributed logarithmically, ranging from $20 \mathrm{~Hz}$ to $1 \mathrm{MHz}$.

\subsubsection{Mohr-type Whipping System}

The whipping machine (Whipped Cream Tester CP-Lift V2.0, Janz-Präzisionstechnik GmbH, Malente/Germany) was designed to whip foams in a controlled manner. The current consumption of the motor during the whipping process is proportional to the energy required to maintain the rotation speed and to the firmness of the foam. Whipping solutions will cause the current to increase with increasing firmness of developing foam. This fact is shown by the auxiliary variable beating number $\mathrm{PE}$. The observed $\mathrm{PE}$

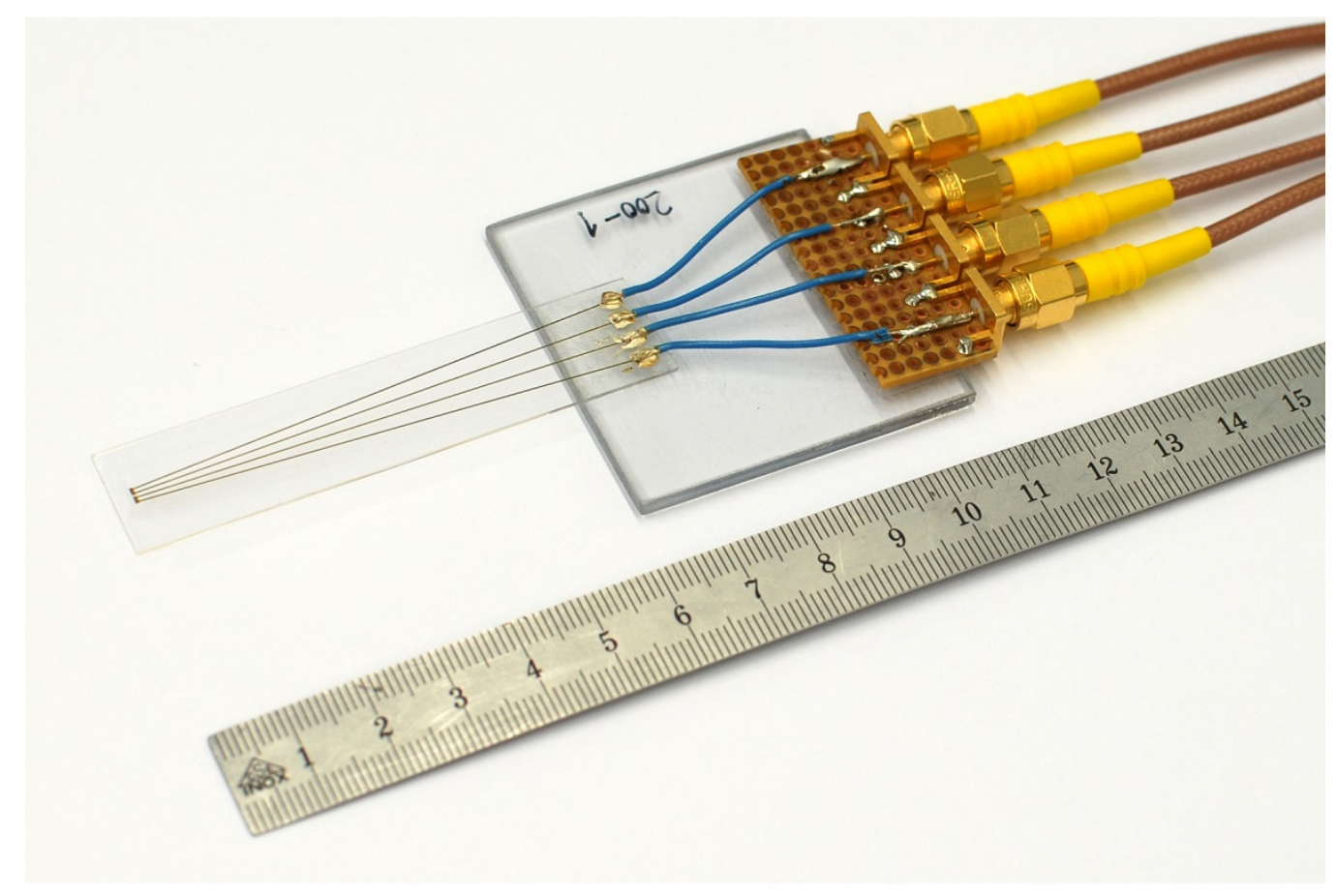

Fig. 2 Measurement device. 


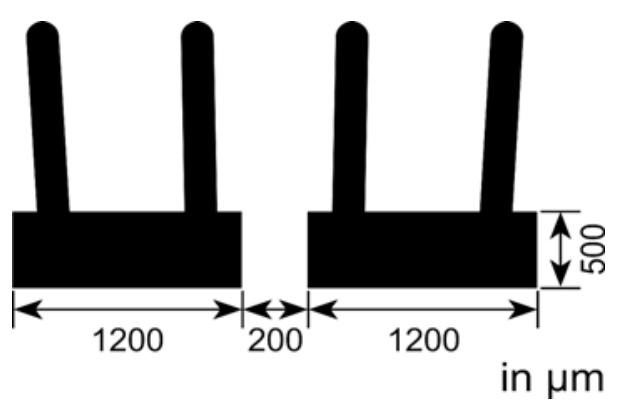

Fig. 3 Schematic close up view of the electrode tip.

values (beating resistance) ranged from 25 to 48 corresponding from liquid to whipped cream with first signs of churning.

2.2.5 Determination of Particle Size Distribution (PSD)

Fat globule sizes in the non-whipped cream and fat globule agglomeration sizes in the whipped cream were determined using a laser diffraction particle size analyzer LS ${ }^{\text {TM }} 13320$ SW (Beckman Coulter, US) with integrated Polarization Intensity Differential Scattering (PIDS) technology. The samples were diluted (1:50) in demineralized water and injected in the analyzer to reach an obscuration level between $8 \%$ and $12 \%$, respectively, $40 \%$ to $60 \%$ for PIDS obscuration. The volumetric PSDs $\mathrm{q} 3$ and distribution parameters were calculated from the intensity profile of the scattered light with Fraunhofer optical model using the instrument's software. All analyses were performed in duplicate.

\section{Results and Discussion}

\subsection{Phase Separation of an Emulsion (Oil-Vinegar)}

When an oil-vinegar mixture is monitored with impedance spectroscopy measurements, it is possible to position the electrodes at different locations with respect to the separated state. This is illustrated in Fig. 4. For one setup, the electrodes are located in the oil region after phase separation (Fig. 4, right side), for the other setup, the electrodes are located in the vinegar region (Fig. 4, left side). For both positions, IS measurements have been done during the separation process of an oil-vinegar mixture at room temperature. The corresponding results are shown in Fig. 5 (electrodes in the oil zone) and Fig. 6 (electrodes in the vinegar zone). From the data shown in Fig. 5 it can be seen that both, phase (Fig. 5a) and amplitude (Fig. 6b) change significantly as a function of time but not at all frequencies. The phase change is most prominent between $10^{4}$ and $10^{5} \mathrm{~Hz}$. In the emulsified state, the phase angles are in the range of 0 degrees, which is typical for ionic conductors. With ongoing separation and increasing frequency, the phase angles become more negative because of the isolating effect of oil.

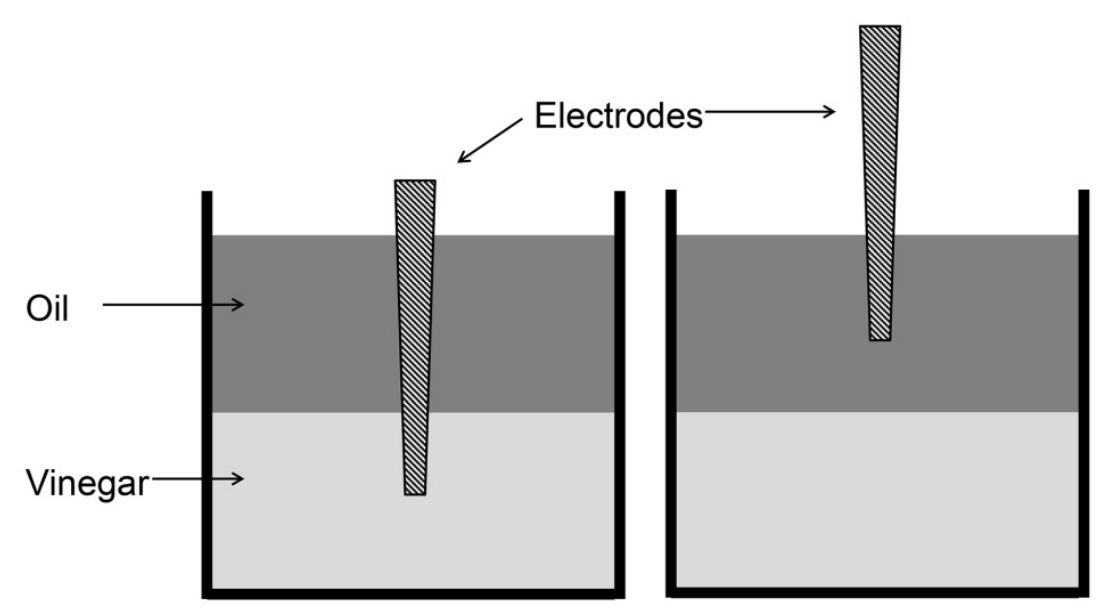

Fig. 4 Possible electrode positions for measuring the oil-vinegar phase separation. The measurement zone is located at the bottom end of the electrode and this zone is several $\mathrm{mm}$ away from the interface region. 

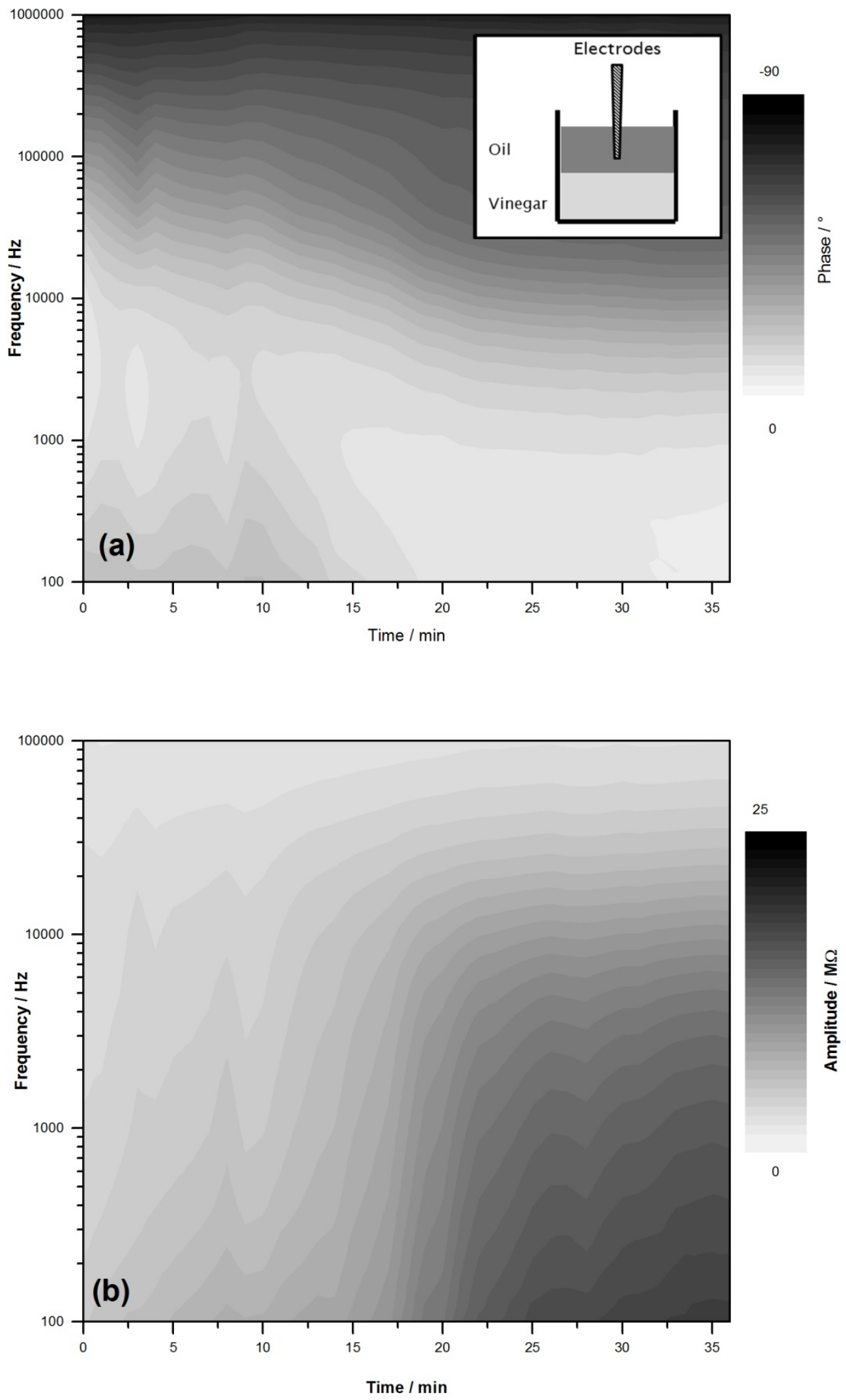

Fig. 5 Time-dependent signal sequence of the phase (a) and amplitude (b) of an IS measurement on phase separating and coalescing an oil-vinegar emulsion. Phase and amplitude values are represented as grey scales. The bipolar electrode is placed so that it was located in the zone of the oil in the phase-separated state (see inset in Fig. 5a). 
Local Impedance Spectroscopy: A Potential Tool to Characterize the Evolution of Emulsions and Foams 255
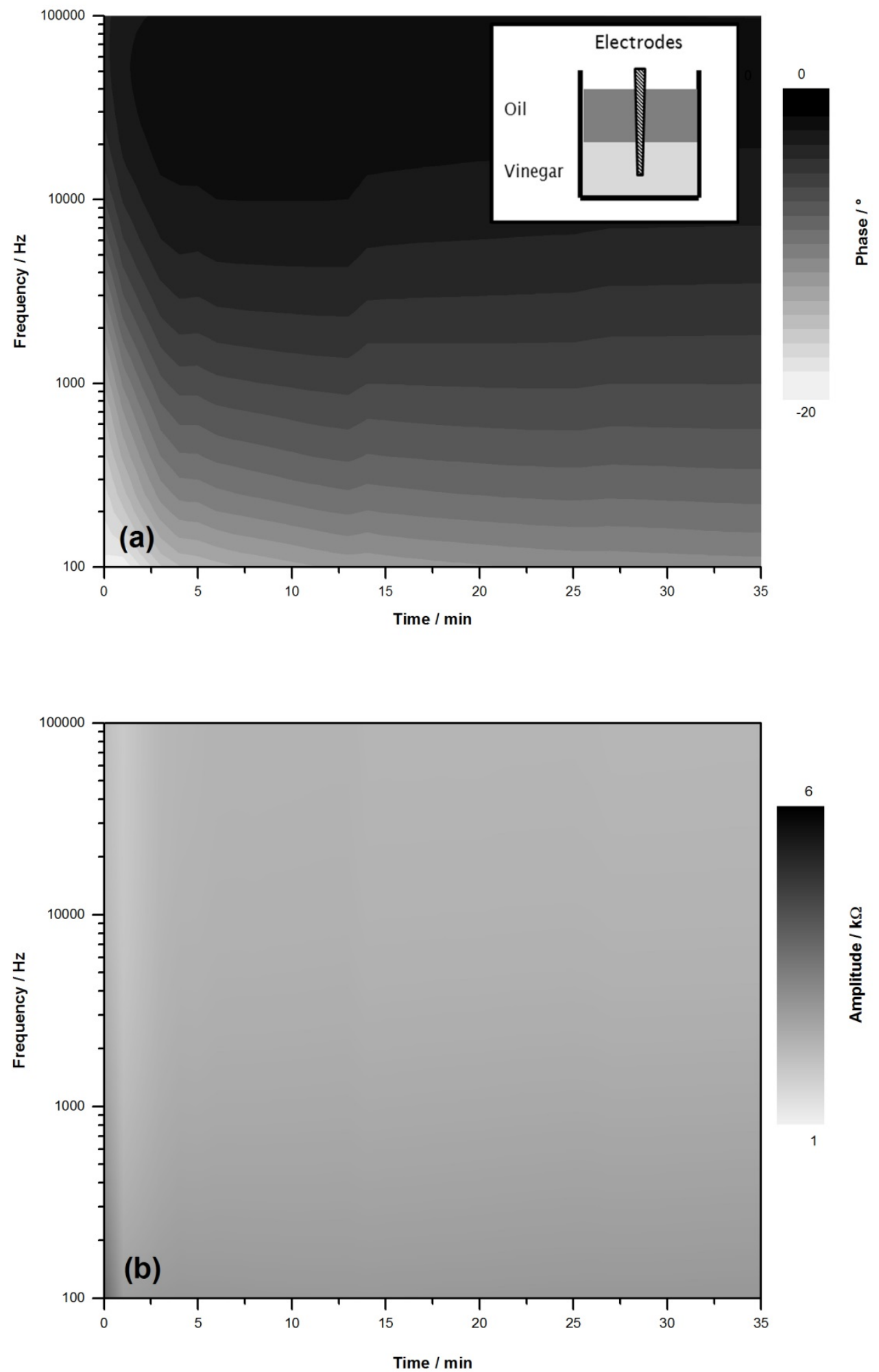

Fig. 6 Time-dependent signal sequence of the phase (a) and amplitude (b) of an IS measurement on an oil-vinegar emulsion. Phase and amplitude values are represented as grey scales. The bipolar electrode is placed so that it was located in the zone of the vinegar in the phase-separated state (see inset in Fig. 6a). 
Amplitude variations on the other hand can be observed below $10^{4} \mathrm{~Hz}$. It can be concluded from the data that the phase separation takes place mainly in the interval between 15 and $20 \mathrm{~min}$. Since the oil is a poor conductor, the values of the amplitude signal are within the $M \Omega$ range and are increasing with time because the measurement region is continuously depleting from charge carriers originating from vinegar. Furthermore, the data also nicely illustrate that a statement about the process of phase separation is only possible due to the measurements over a large frequency range.

A measurement on an identical sample but with the measuring electrodes located in the zone of the vinegar (after phase separation) yields the measurement data, which are shown in Fig. 6.

It is obvious that no significant statement about the phase separation can be made from this data set. The ions, which contribute to the conductivity, are provided by the vinegar. If a vinegar-rich zone is depleted from oil (no contribution to the conductivity) then only minor changes in the IS signals can be expected, i.e. the separation process cannot be monitored.

Because charge carriers are provided by the vinegar, the amplitude values for the measurement shown in Fig. $6 \mathrm{~b}$ are about a magnitude smaller than for the data shown in Fig. 5b. In the low frequency range at the beginning separation process, one may observe slightly lower phase angles than towards the end of the separation process which is due to the isolating behavior of oil. The presented results on the phase separation of oil and vinegar show on the one hand the potential for measuring time-dependent processes by IS (even though not necessarily for systems like oil and vinegar), on the other hand our experiments also show that a careful experimental setup (e.g. position of the electrodes) is necessary to obtain the desired information. The advantage gained by measuring locally is that due to the small probed volume of about $1 \mathrm{~mm}^{3}$, that also minor changes in the charge carrier concentration can be monitored and, as a consequence, the time resolution of the measurement increases.

Fig. 7 compares the impedance values of pure vinegar (black solid line) and pure oil (black dashed line) with the vinegar (triangles) and oil (circles) measured after a time of $35 \mathrm{~min}$ for the separation process. After this time the two components were fully separated as observed through visual inspection. After 35 min of separation the vinegar region will still contain some oil, and the oil region will still contain some vinegar, even though not visible by eye. As can be seen from Fig. 7, the curves measured in pure vinegar and in the vinegar zone after separation are almost congruent. Due to the fact that the impedance signal is ruled by the vinegar charge carriers, some oil remnants in the vinegar of the phase separated zone will not influence the measured data. The situation is different if the impedance of pure oil and oil in the phase separated state are compared. Here, the oil in the phase separated state yields impedance which is about three magnitudes lower than the corresponding values for pure oil. The reduced impedance is caused by small amounts of remaining vinegar in the oil zone after separation.

This behavior implies that the method seems to be suited to measure small amounts of water-based liquids containing charge carriers. Apotential application field could be the monitoring of the drying process of fats.

\subsection{Examination of the Whipping Process of Cream}

In the previous chapter we presented a system where a separation process was monitored. As a second test system to investigate the potential of local IS, the whipping-induced conversion of an emulsion (dairy cream) into a fat foam was investigated and IS results compared to data from PSD measurements. In contrast to the above discussed o/w-emulsion, cream as an emulsion can be whipped by the incorporation of a gas phase (usually air), so that the 2-phase system "emulsion" turns into a 3-phase system "foam" [15]. 


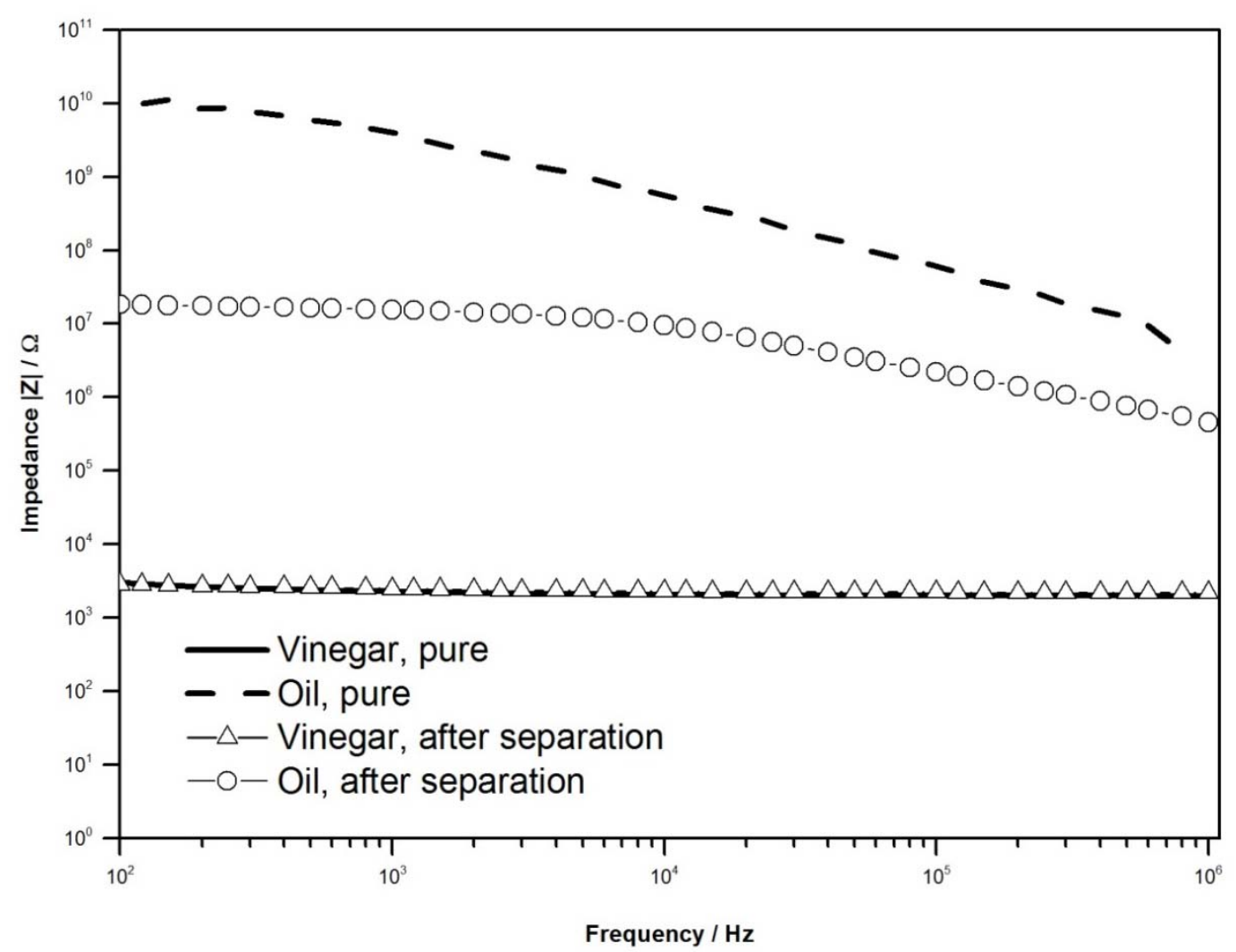

Fig. 7 Frequency dependent impedance of pure vinegar (solid line), pure oil (dashed line), vinegar after separation (triangles) and oil after separation (circles).

More particular, whipped cream can be regarded as foam stabilized by fat globules. This stabilization takes place as the fat globules present in the cream move to the gas-water interface during whipping [16].

A prerequisite for the development of a stable fat globule network incorporating air bubbles is the partial crystallinity of the fat globules. A widely accepted model suggests that such partially crystallized globules have a shell of crystallized fat and a center with fluid fat as with cooling the milk fat is fractionated according to the crystallization temperatures of the different fat fractions. When whipped, a part of the globules is damaged and provides fluid fat to act as a binder material for either intact fat or crystalline fragments of broken fat globules [17].

The whipping process of cream can be separated into different steps. Within the first period, air bubbles are incorporated into the emulsion. The first stabilization is given by milk protein (casein micelles, whey proteins) which leads to a strong volumetric increase of the system [18]. After this initial stabilization, fat globules start to move to the air-water interface, replacing the protein there. Approaching the interface, the primary membrane of the fat globules is displaced from the fat globules as the globules start to protrude into the air phase [19]. Contemporarily with the orientation of the fat globules at the interface and due to the mechanical energy input, a part of the fat globules is damaged in such a way that liquid fat is released. This fat then can act as a binder material to agglomerate the loosely orientated intact fat globules at the interface and forming stable fat globules shells around the air bubbles. Furthermore, a three-dimensional network of stabilized air bubbles can be formed giving overall stability to the whipped cream $[17,20]$. 
Overruns for non-homogenized, whipped cream typically are in the range of $100 \%$ to $150 \%$. Whipped cream can be considered as a 3-phase system, in which air represents the inner dispersed phase, incorporated into a three-dimensional network of agglomerated fat globules which is surrounded by the water phase. Milk proteins (approx. 3.3\%, w/w) as well as original membrane material from the fat globules, lactose (approx. 4.6\%, w/w), milk salts (approx. 0.8\%, w/w) and further minor milk components are dispersed or dissolved in the water phase [21].

In Fig. 8a, the PSD (giving the percentage of volume which the particles occupy at the detected diameter on the $y$-axis) as function of the PE-values is represented in a grey-scale image. Only diameters below $12.4 \mu \mathrm{m}$ are considered. This threshold value was chosen as it represents the particle size maximum of the native, unbeaten cream. The formation of fat globule aggregates decreases the quantity of non-aggregated native fat globules, thus the formation of aggregates can be monitored directly (measurement range of the native fat globules and above $12.4 \mu \mathrm{m}$ for the agglomerates) or indirectly (measurement range below $12.4 \mu \mathrm{m}$, monitoring only the native fat globules). However, if fat globule agglomerates are formed above a certain size, they start to separate quickly to the sample surface and it is impossible to include them in the particle size measurements. So the relation of large agglomerates to small agglomerates and native globules as measured in the instrument shifts to smaller object sizes. As a consequence, the quantity of particles below $12.4 \mu \mathrm{m}$ apparently could increase on the paper again in the later whipping stages.

One can deduct from this graph four distinct sections: First section with the native particle size distribution, second section with a volumetric reduction of this particle fraction and a shift towards smaller diameters and a third and fourth section in which the PSD volumetrically increases and decreases nearly completely again. To highlight, four sections are separated in the graph by vertical dashed lines.

Fig. $8 \mathrm{~b}$ shows the phase signal of the local IS measurements of cream as a function of the PE-values for frequencies between $10^{4}$ and $10^{6} \mathrm{~Hz}$. The distinctive changes observed in the PSD as function of the PE-values are again marked by dashed vertical lines. One can see that these special PE-values can also be inferred from the IS data.

Both measurement methods therefore yield similar information regarding relevant $\mathrm{PE}$-values for the cream whipping process. It is known from literature [22] and own research that cream passes different stages during whipping with respect to microstructure development. In the first 60-80 s of whipping, air is basically incorporated in the emulsion. This early stage has not been time-resolved with the measurements presented here and will be subject of further studies. Although we have not investigated it in detail, it is our impression that the four sections as well as the phase angle maxima indicate the domination of different microstructural regimes in the whipped cream or situations in which the domination of a microstructural aspect is replaced by another one. A further and much more detailed examination of the linkage of phenomenological properties of cream, the beating resistance over the whipping time and correlations as well as the deduction of the potential of IS to elucidate microstructures in cream will be done in a following paper.

From our point of view, IS to monitor the whipping process also has a practical impact. PSD measurements are time consuming and phase separation (large agglomerates, probably linked to air bubbles) of the samples complicates to perform measurements really comprising the whole sample information. IS measurements on the other hand are easy, do not require sample preparation before measurement and are, with a measurement time of less than $1 \mathrm{~min}$, also considerably faster. In addition, it is in principle also possible to integrate IS micro-electrodes 

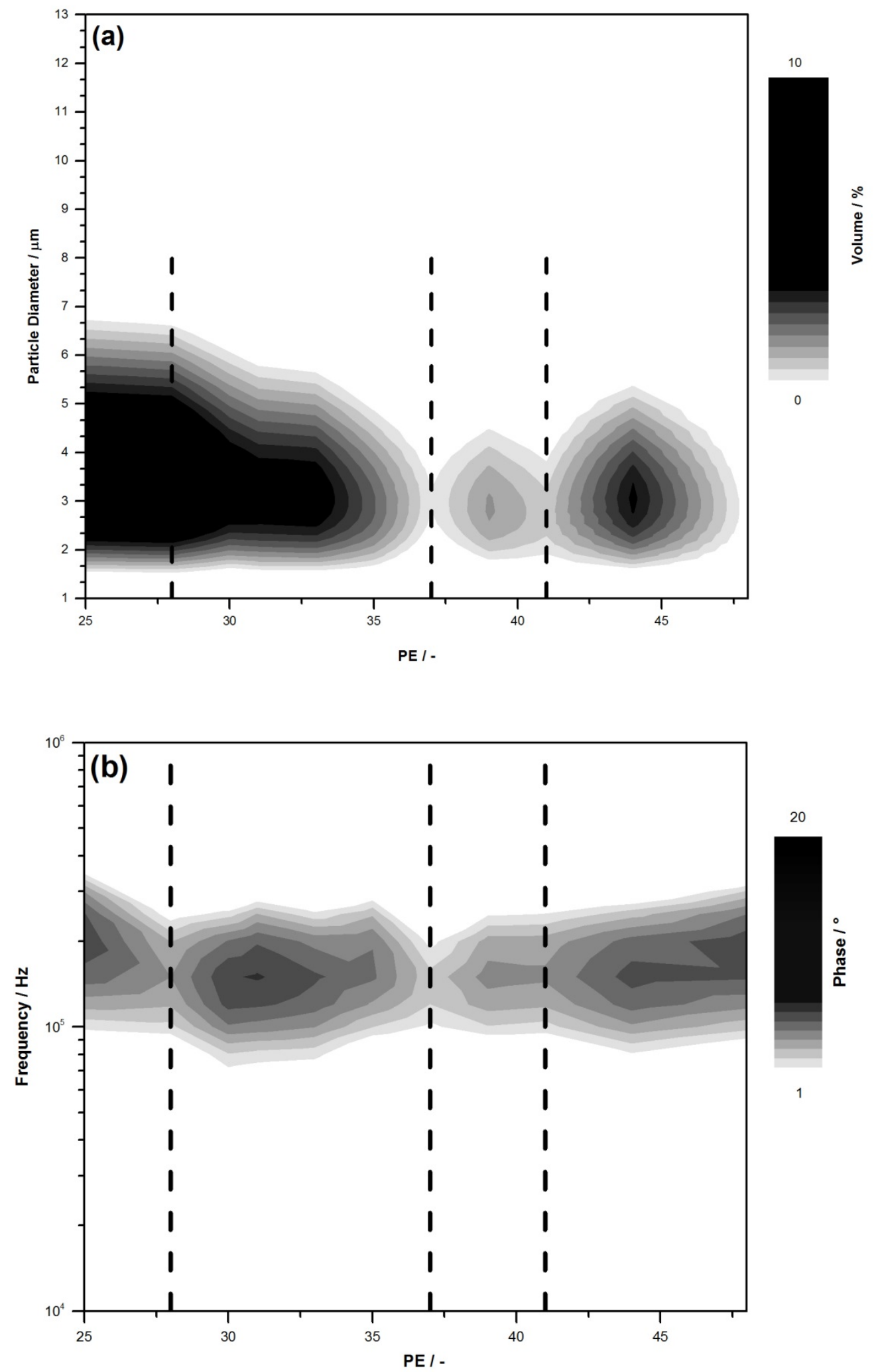

Fig. 8 PSD from light scattering experiments (a) and phase signal from the local IS measurement; (b) as a function of the beating number PE. The vertical dashed lines mark PE values with distinctive changes in the cream. 
into a whipping machine to monitor as well as to control the whipping process in-situ and in quasi-real-time.

Possible limitations and difficulties of local IS: Because different samples all bring their own challenges, we restrict ourselves to some general statements. In impedimetric measurements of electrolytes special attention needs to be paid to the temperature. The temperature dependence is mainly caused by an increased viscosity but also by a raised ion-concentration [23]. Further, stirred/moving media disturb the formation of the Gouy-Chapman layer originated from the diffusion process of the charge carriers [13]. We have observed a slight browning of the electrodes with consecutive measurements. Protein adsorption occurs within the first few seconds of electrode-protein contact, and is an irreversible process [24]. We assume that the browning of the electrodes originates from this protein adsorption. With reference measurements using $10 \%$ citric acid solution before and after the measurements we did not observe any change of the electrical behavior of the electrodes. Therefore we conclude that the browning of the electrodes does not influence the measurements.

\section{Conclusions}

In this paper the potential of local IS measurements as a tool for the characterization of processes involving emulsions and dairy foams has been investigated. It could be shown that the separation process of two liquids (oil and vinegar) can be monitored in real time. However, one has to pay attention to the configuration of the measuring electrodes, since already few ions in a non-conductive component of an emulsion can disturb the impedance signal significantly.

Furthermore, we found first indications that IS measurements could be a valuable tool to monitor the evolution of fat foams as it seems to detect similar microstructural aspects/changes compared to a more common technique. We found a good correlation between the local IS and the PSD for a selected size range measured by light scattering over the whole whipping process. This circumstance demonstrates the ability of the local IS to quickly access and monitor modifications of the microstructure (whipping cream), temporal changes (separating emulsion) or the addition of foreign substances (small amounts of water in oil).

\section{Acknowledgements}

This work has been financially supported by an armasuisse $\mathrm{W}+\mathrm{T}$ grant.

\section{References}

[1] Awad, T. S., Moharram, H. A., Shaltout, O. E., Asker, D., and Youssef, M. M. 2012. "Applications of Ultrasound in Analysis, Processing and Quality Control of Food." A Review, Food Research International 48: 410-27.

[2] Gowen, A. A., Tiwari, B. K., Cullen, P. J., McDonnell, K., and O'Donnell, C. P. 2010. "Applications of Thermal Imaging in Food Quality and Safety Assessment." Trends in Food Science \& Technology 21 (4): 190-200.

[3] Huang, H., Yu, H., Xu, H., and Ying, Y. 2008. "Near Infrared Spectroscopy for On/in-line Monitoring of Quality in Foods and Beverages." A review, Journal of Food Engineering 87 (3): 303-13.

[4] Olafsdottir, G. 2004. "Multisensor for Fish Quality Determination." Trends in Food Science \& Technology 15 (2): 86-93.

[5] Pliquett, U. 2010. "Bioimpedance: A Review for Food Processing." Food Engineering Reviews 2: 74-94.

[6] Kent, M. 1975. "Time Domain Measurements of the Dielectric Properties of Frozen Fish." Journal of Microwave Power 10: 37-48.

[7] Guermazi, M., Tröltzsch, U., Kanoun, O., and Derbel, N. 2011. "Assessment of Beef Meat Aging Using Impedance Spectroscopy." In 8th International Multi-Conference on Systems, Signals and Devices, 1-6.

[8] Damez, J. L., Clerjon, S., Abouelkaram, S., and Lepetit, J. 2008. "Electrical Impedance Probing of the Muscle Food Anisotropy for Meat Ageing Control." Food Control 19: 931-9.

[9] Scandurra, G., Tripodi, G., and Verzera, A. 2013. "Impedance Spectroscopy for Rapid Determination of Honey Floral Origin.” Journal of Food Engineering 119 (4): 738-43. 
[10] Rehman, M., Abu Izneid, B. A., Abdullah, M. Z., and Arshad, M. R. 2011. "Assessment of Quality of Fruits using Impedance Spectroscopy.” International Journal of Food Science and Technology 46 (6): 1303-9.

[11] Grossi, M., Lanzoni, M., Pompei, A., Lazzarini, R., Matteuzzi, D., and Riccò, B. 2008. "Detection of Microbial Concentration in Ice-Cream Using the Impedance Technique.” Biosensors and Bioelectronics 23 (11): 1616-23.

[12] Grossi, M., Lazzarini, R., Lanzoni, M., and Riccò, B. 2011. "A Novel Technique to Control Ice Cream Freezing by Electrical Characteristics Analysis.” Journal of Food Engineering 106 (4): 347-54.

[13] Pliquett, U. 2010. "Testing Miniaturized Electrodes for Impedance Measurements within the $\beta$-dispersion-A Practical Approach." Journal of Electrical Bioimpedance 1 (1): 41-55.

[14] Bates, J. B., and Chu, Y. T. 1992. "Electrode-Eiectrolyte Interface Impedance: Experiments and Model." Annals of Biomedical Engineering 20: 349-62.

[15] Kessler, H. 2002. Food and Bio Process Engineering-Dairy Technology.München: Verlag A. Kessler, 694.

[16] Tamime, A. 2007. Structure of Dairy Products. Oxford, UK: Blackwell Publishing Ltd., 288.

[17] Fredrick, E., Walstra, P., and Dewettinck, K. 2010. "Factors Governing Partial Coalescence in Oil-in-Water Emulsions." Advances in Colloid and Interface Science
153 (1-2): 30-42.

[18] Walstra, P., Geurts, T. J., and Wouters, J. T. M. 2006. Dairy Science and Technology. Boca Raton: CRC/Taylor \& Francis, 782.

[19] Hotrum, N. E., Stuart, M. A. C., van Vliet, T., Avino, S. F., and van Aken, G. A. 2005. "Elucidating the Relationship between the Spreading Coefficient, Surface-Mediated Partial Coalescence and the Whipping Time of Artificial Cream." Colloids and Surfaces A: Physicochemical and Engineering Aspects 260 (1-3): 71-8.

[20] Goff, H. D. 1997. "Instability and Partial Coalescence in Whippable Dairy Emulsions.” Journal of Dairy Science 80 (10): 2620-30.

[21] Töpel, A. 2016. Chemie und Physik der Milch: Naturstoff, Rohstoff, Lebensmittel. Hamburg: Behr's Verlag, 738.

[22] van. Aken, G. A. 2001. "Aeration of Emulsions by Whipping." Colloids and Surfaces A: Physicochemical and Engineering Aspects 190: 333-54.

[23] Wagner, H. 2012. Einfluss der Temperatur auf die elektrische Leitfähigkeit verdünnter, wässriger Lösungen, VGB POWERTECH, 82-9.

[24] Moulton, S. E., Barisci, J. N., Bath, A., Stella, R., and Wallace, G. G. 2003. "Investigation of Protein Adsorption and Electrochemical Behavior at a Gold Electrode." Journal of Colloid and Interface Science 261 (2): 312-9. 